\title{
Neurodevelopmental Disorders: The Journey, the Dreams and their Realization
}

\author{
Sheffali Gulati \\ Child Neurology Division, Department of Pediatrics, \\ All India Institute of Medical Sciences, New Delhi.
}

\begin{abstract}
Neuro Developmental Disorders (NDDs) are associated with significant morbidity. This involves early identification of the disorder, the correct management of the disorder and associated disabilities. In India, the paucity of trained personnel and lack of knowledge about these disorders has been instrumental in inadequate management and recognition of these NDDs. The Child Neurology Division, Department of Pediatrics at All India Institute of Medical Sciences has made few noteworthy and meaningful contributions in these aspects: devising a DM curriculum for pediatric neurology, developing indigenous tools for diagnosing these NDDs and performing relevant research. These endeavors would go a long way in serving the children with NDDs.
\end{abstract}

Keywords: Neurodevelopmental disorders, autism, cerebral palsy, attention deficit hyperactivity disorder.

\section{Introduction}

Neuro Developmental Disorders (NDDs) constitute a significant proportion of morbidity handled by pediatric health care services. These include cerebral palsy, autism, attention deficit hyperactivity disorder (ADHD) and intellectual disability, epilepsy, autoimmune disorders including autoimmune encephalitis and neuromuscular disorders (including muscular dystrophy). A recent study estimated prevalence of 'any NDD' in 2-9 year old children in India to be $12 \cdot 0 \%$ (95\% CI: $10 \cdot 9-13 \cdot 2 \%)$, and $21 \cdot 8 \%$ of these had more than one NDD (unpublished data). Thus, neurological disorders represent a significant cause of morbidity among children.
In addition, there has been a paucity of physicians trained in pediatric neurology and also, inadequate research focusing on disorders specific to our nation, which has further compromised care of these children. Hence, to overcome these lacunas, the Child Neurology Division, All India Institute of Medical Sciences has taken enormous steps over the past several years. These have been highlighted in subsequent sections.

\section{DM Pediatric Neurology}

In order to overcome the scarcity of physicians trained in Pediatric Neurology in our country, Pediatric Neurology program was

Correspondence : Prof. Sheffali Gulati, Chief, Child Neurology Division, Department of Pediatrics, All India Institute of Medical Sciences, New Delhi-110029.

DR. S. JANAKI MEMORIAL ORATION delivered during NAMSCON 2016 at the All India Institute of Medical Sciences, Raipur. 
initiated in 2004, at All India Institute of Medical Sciences, under the Child Neurology Division as a part of Department of Pediatrics. Over the past 12 years, twenty-five residents have qualified as DM Pediatric Neurology. These residents are subjected to rigorous training under all aspects of clinical care as well as neuroradiology, neuropathology and other clinical and rehabilitative aspects of child neurology. The DM program at AIIMS has contributed to trained manpower not only in our country, but has also trained fellows from other SAARC nations, United States of America, France, and United Kingdom.

\section{Research}

There has been scarcity of research in pediatric neurological disorders from our country. Numerous national and international research projects have been undertaken at the Child Neurology Division, AIIMS, which contributed to the arena of epilepsy, autism, other NDD (like cerebral palsy), and autoimmune disorders. The division has been a pioneer in the country in developing dietary therapies for drug resistant epilepsy (1). The children whose epilepsy fails to be adequately controlled despite two antiepileptic drugs are defined to have drug resistant epilepsy (2). The

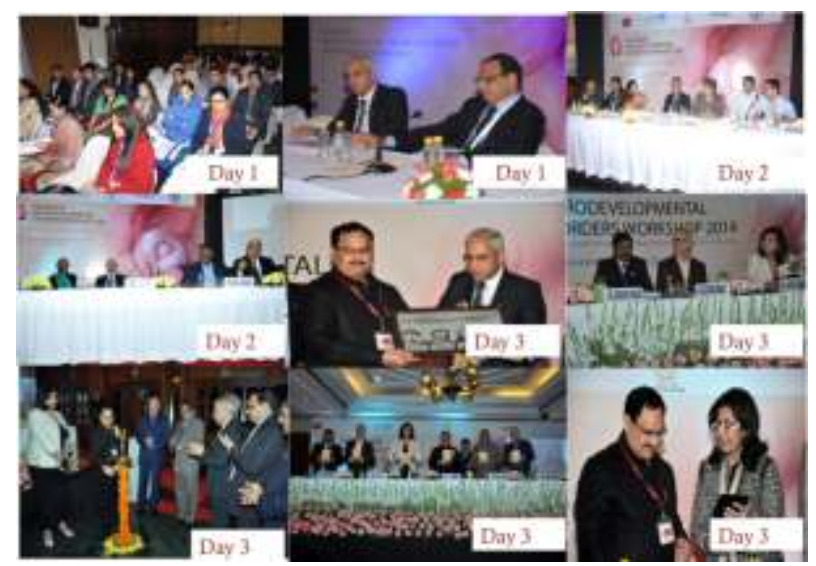

Fig. 1: Neurodevelopmental Disorders workshop was organized in December 2014. The picture shows the different events during the workshop. therapeutic options for these children include epilepsy surgery or dietary therapy. The division has demonstrated the efficacy of these dietary therapies in drug resistant epilepsies using ketogenic diet, modified Atkins diet, and low glycemic index dietary therapy (3-7). In addition, it has contributed to the development of 2.5:1 ketogenic diet and proved its efficacy in institutional research (3). The division also, has been the frontrunner in diagnosing, managing and optimizing the treatment for autoimmune encephalitis (8), leukodystrophies (9), and acquired demyelinating disorders of CNS (10). Also, the research focusing on domiciliary management of intranasal and buccal midazolam has helped to standardize the management protocols for seizures at home (11). These researches have been instrumental in developing various therapeutic guidelines, and have also paved the way for various national programs including Rashtriya Bal Swasthya Karyakram (RBSK) (12).

\section{Public Health Initiatives}

Educating the parents and children and the society at large is an important aspect about management of any disease. The Child Neurology Division has played a pivotal role in educating the society about NDDs (Fig. 1),

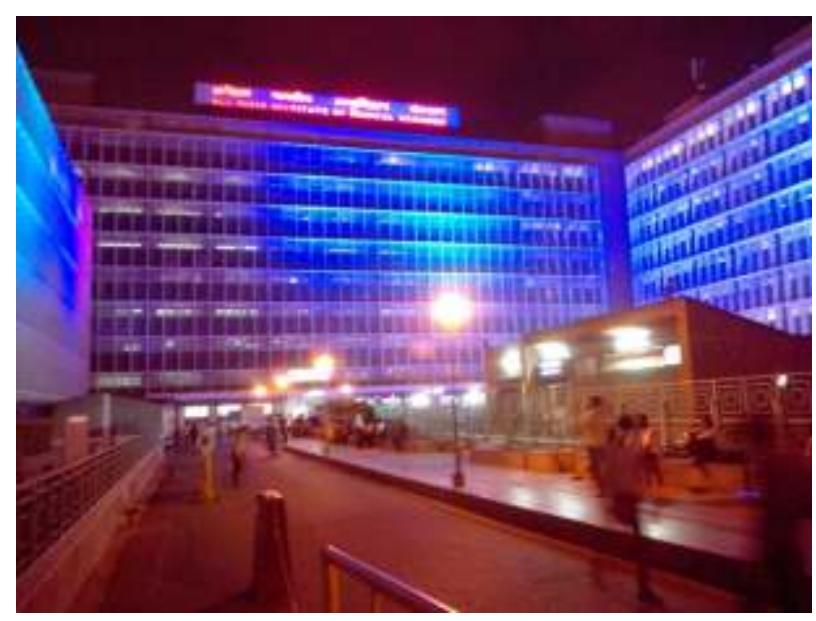

Fig. 2 : The building of AIIMS, New Delhi being lit blue on 30 Apr 2015 to commemorate Autism Day. 


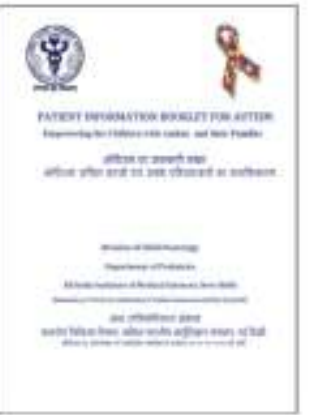

2014 Empowering the children with autism and their
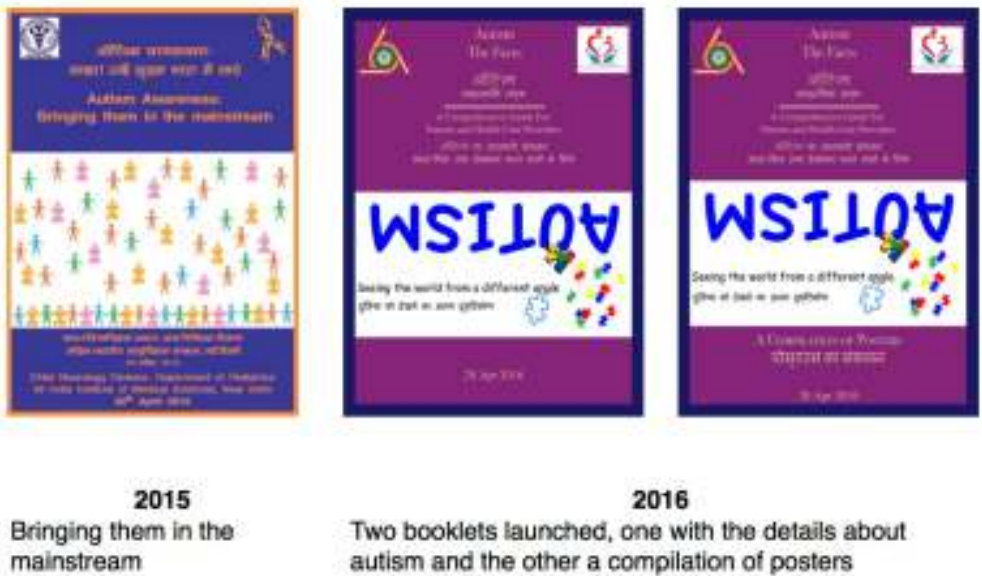

2016

Two booklets launched, one with the details about autism and the other a compilation of posters

Fig. 3 : Autism-related patient information booklets launched by Child Neurology Division, Department of Pediatrics, AIIMS

autism (Fig. 2 to 4 ), and epilepsy by organizing regular public health lectures, television and radio shows/ interviews, newspaper interviews and information materials. In addition, the division has published patient educational booklets on all the common pediatric neurological disorders. These are freely d o w n l o a d a b l e f r o m http://www.aiims.edu/en/2014-12-24-07-1628/neurology_educatio.html (Fig. 3) (13). Also the division has launched a helpline (with mobile number 9868399037 and email: autismhelp.pedsaiims@gmail.com, pedneuroaiims@yahoo.com) to facilitate help to parents of sick children.

\section{Assisting Government Initiatives}

The division was part of the INCLEN Collaborative study (with me being the Network Coordinator and Site PI), which estimated the prevalence of various neurological disorders across the country (14-17). This was instrumental in developing the convergence framework of RBSK (12). Also, the division has worked in close liaison with the Government of India for the certification of autism as a disability and issue of certificate for this disorder. The division in close collaboration with Ministry of Social Justice \& Empowerment, Government of India, organized the workshop for training the master trainers for diagnosis of autism across the country in August- September 2016. This was aimed at formulating a uniform instrument for diagnosis of autism, and training health care professionals to use that tool and spreading awareness about the certification process.

\section{Innovation}

The division has been significantly contributed to several decisive innovations. These include use of skin biopsy to diagnose muscular dystrophy (especially collagen 6 related disorders) (18-20). Also, the division has revolutionized telephonic follow-up for disorders like neurocysticercosis, LennoxGastaut Syndrome, and Duchenne Muscular Dystrophy.

The division has also been pivotal in developmental of tools for diagnosis of various NDDs. Initially these tools were developed in collaboration with INCLEN (14-17). The tools 


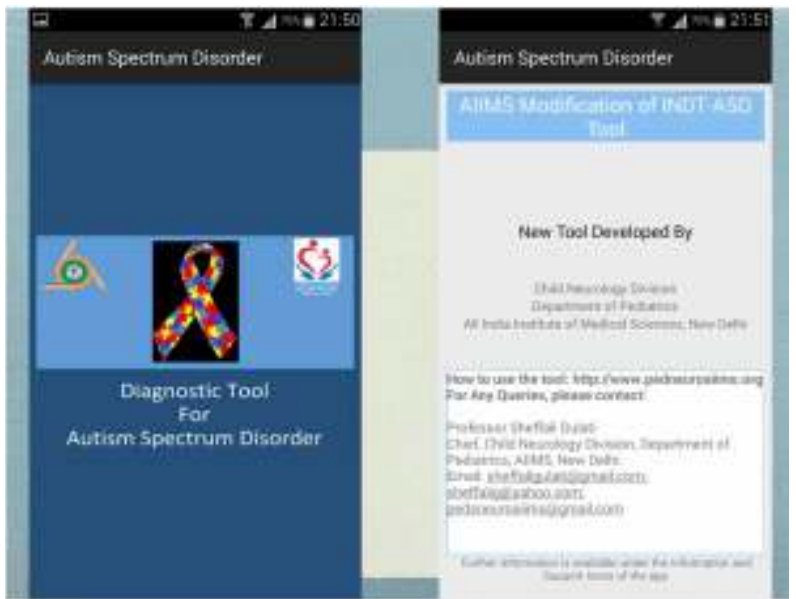

Fig. 4 : Snapshot of mobile app on Autism launched by Child Neurology Division, Department of Pediatrics, AIIMS on 28 Apr 2016

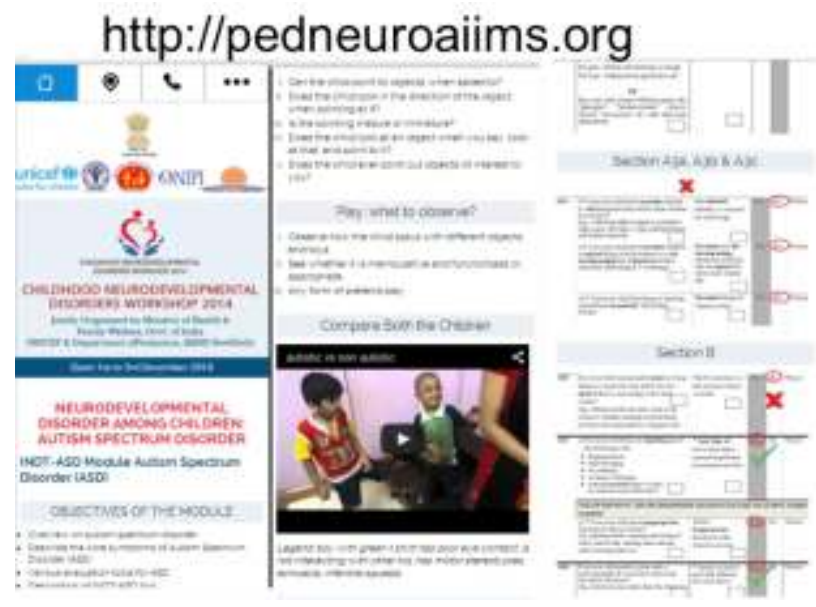

Fig. 5 : Snapshot of microsite, with autism related information, launched by Child Neurology Division, Department of Pediatrics, AIIMS

were later updated as per the latest guidelines. These AIIMS modified INDT tools for diagnosis of epilepsy, neuromuscular impairment, ADHD, and autism have been validated in institutional research. The tools for Autism Spectrum Disorder (https://play.google.com/ store/apps/details?id=app.autism.sanyakhurana diagnostictoolforautismspectrumdisorder and https://itunes.apple.com/us/app/autism- s p e c trum - disorder-diagnostic/ id $1151524697 ? \mathrm{mt}=8$ ) and epilepsy (https://play.google.com/store/apps/details?id= com.weboutsourcing.childhoodepilepsy.childh oodepilepsydiagnostictool\&hl=en and https://itunes.apple.com/in/app/childhoodepilepsy-diagnostic/id1078173463? $\mathrm{mt}=8$ ) have been converted into a mobile application (Fig. 4) which is downloadable free of cost, while applications for other tools are under development.

\section{Microsite}

The Child Neurology Division of Department of Pediatrics at AIIMS launched a m o b i l e - b a s e d w e b s i t e (m.nddworkshop2014.org) on 03-December2014. This microsite was renamed as m.pedneuroaiims.org, in Dec 2015. This microsite deals in NDD including ADHD, Autism Spectrum Disorders, Intellectual Disability, Epilepsy, Learning Disability, Neuromuscular Disorders, Cerebral Palsy, Speech and Language Disorders, Hearing, and Vision Impairment. The microsite has various educational materials including videos, for doctors for clinical applications and for parents for home-based intervention (Fig. 5).

\section{Future}

The various initiatives mentioned above have laid the foundation for a healthier child neurology environment, where we can expect evidence-based management of neurological disorders among children. To build on these foundations, it is planned to perform high quality research covering all domains of neurology in collaboration with international leaders.

\section{Conclusion}

Neurological disorders are an enormous burden in terms of associated disabilities. In association most of these disorders are chronic and require patience and expertise for management. Various measures including 
training physicians, research, innovations and public health initiatives will immensely contribute in improving the management of these children individually and also educate the society about the rights of these disabled children.

\section{References}

1. Sharma S, Gulati S, Kalra V, Agarwala A, Kabra M (2009). Seizure control and biochemical profile on the ketogenic diet in young children with refractory e pilepsy-Indian experience. Seizure18(6):446-449.

2. Kim JA, Yoon JR, Lee EJ, et al (2016). Efficacy of the classic ketogenic and the modified Atkins diets in refractory childhood epilepsy. Epilepsia 57(1):5158.

3. Raju KN, Gulati S, Kabra M, et al (2011). Efficacy of 4:1 (classic) versus 2.5:1 ketogenic ratio diets in refractory epilepsy in young children: a randomized open labeled study. Epilepsy Res 96(1-2):96100.

4. Sharma S, Gulati S (2012). The ketogenic diet and the QT interval. $J$ Clin Neurosci 19(1):181-182.

5. Sharma S, Sankhyan N, Gulati S, Agarwala A (2012). Use of the modified Atkins diet in infantile spasms refractory to first-line treatment. Seizure 21(1):4548.

6. Sharma S, Sankhyan N, Gulati S, Agarwala A (2013). Use of the modified Atkins diet for treatment of refractory childhood epilepsy: a randomized controlled trial. Epilepsia 54(3):481-486.
7. Sharma S, Jain P, Gulati S, Sankhyan N, Agarwala A (2015). Use of the modified Atkins diet in Lennox Gastaut syndrome. JChild Neurol30(5):576-579.

8. Pandit AK, Ihtisham K, Garg A, Gulati S, Padma MV, Tripathi M (2013). Autoimmune encephalitis: A potentially reversible cause of status epilepticus, epilepsy, and cognitive decline. Ann Indian Acad Neurol 16(4):577-584.

9. Gulati S, Jain P, Chakrabarty B, Kumar A, Gupta N, Kabra M (2016). The spectrum of leukodystrophies in children: Experience at a tertiary care centre from North India. Ann Indian Acad Neurol 19(3):332-338.

10. Gulati S, Chakrabarty B, Kumar A, et al (2015). Acquired demyelinating disorders of central nervous system: A pediatric cohort. Ann Indian Acad Neurol 18(Suppl 1):S48-S55.

11. Bhattacharyya M, Kalra V, Gulati S (2006). Intranasal midazolam vs rectal diazepam in acute childhood seizures. Pediatr Neurol 34(5):355-359.

12. Anonymous. (2014). Setting up district early intervention centres: Operational Guidelines. Rashtriya Bal Swasthya Karyakam, Child Health Screening and Early Intervention Services under NHM. New Delhi: Ministry of Health \& Family Welfare, GoI.

13. Anonymous (2014). Patient Education Booklet. New Delhi : All India Institute of Medical Sciences.

14. Gulati S, Aneja S, Juneja M, et al (2014). INCLEN Diagnostic Tool for Neuromotor Impairments (INDT-NMI) for primary care physician: development and validation. Indian Pediatr 51(8):613-619. 
15. Juneja M, Mishra D, Russell PS, et al (2014). INCLEN Diagnostic Tool for Autism Spectrum Disorder (INDT-ASD): development and validation. Indian Pediatr 51(5):359-365.

16. Konanki R, Mishra D, Gulati S, et al (2014). INCLEN Diagnostic Tool for Epilepsy (INDT-EPI) for primary care physicians: development and validation. Indian Pediatr 51(7):539-543.

17. Mukherjee S, Aneja S, Russell PS, et al (2014). INCLEN diagnostic tool for attention deficit hyperactivity disorder (INDT-ADHD): development and validation. Indian Pediatr 51(6):457-462.
18. Tanveer NO, Sharma MC, Sarkar C, et al (2009). Diagnostic utility of skin biopsy in dystrophinopathies. Clin Neurol Neurosurg111(6):496-502.

19. Chakrabarty B, Sharma MC, Gulati S, et al (2014). Skin biopsy: a new tool to diagnose sarcoglycanopathy. $J$ Child Neurol 29(8):NP5-8.

20. Chakrabarty B, Sharma MC, Gulati S, et al (2014). Dystrophinopathy diagnosis made easy: skin biopsy, an emerging novel tool. JChildNeurol 29(4):469-474. 